\title{
Magnesium Force Fields for OPC Water with Accurate Solvation, lon-Binding, and Water-Exchange Properties: Successful Transfer from SPC/E
}

\author{
Kara K. Grotz ${ }^{1}$ and Nadine Schwierz ${ }^{1}$ \\ Department of Theoretical Biophysics, Max-Planck-Institute of Biophysics, Frankfurt am Main, \\ Germany.
}

(*Electronic mail: nadine.schwierz@biophys.mpg.de)

(Dated: 7 December 2021)

\begin{abstract}
Magnesium plays a vital role in a large variety of biological processes. To model such processes by molecular dynamics simulations, researchers rely on accurate force field parameters for $\mathrm{Mg}^{2+}$ and water. OPC is one of the most promising water models yielding an improved description of biomolecules in water. The aim of this work is to provide force field parameters for $\mathrm{Mg}^{2+}$ that lead to accurate simulation results in combination with OPC water. Using twelve different $\mathrm{Mg}^{2+}$ parameter sets, that were previously optimized with different water models, we systematically assess the transferability to OPC based on a large variety of experimental properties. The results show that the $\mathrm{Mg}^{2+}$ parameters for SPC/E are transferable to OPC and closely reproduce the experimental solvation free energy, radius of the first hydration shell, coordination number, activity derivative, and binding affinity toward the phosphate oxygen on RNA. Two optimal parameter sets are presented: MicroMg yields water exchange in OPC on the microsecond timescale in agreement with experiments. NanoMg yields accelerated exchange on the nanosecond timescale and facilitates the direct observation of ion binding events for enhanced sampling purposes.
\end{abstract}

\section{INTRODUCTION}

Molecular dynamics simulations rely on accurate force field parameters for biomolecules, water molecules, and ions. It seems tempting to combine the force fields of the most promising water models with the most successful ion force field in order to utilize the strengths of each parameter set. However, even for simple cations, the transferability of the ion parameters to different water models is limited. Different water models have a significant effect and can alter the thermodynamic and kinetic properties of the electrolyte solution considerably ${ }^{1-3}$. It is therefore crucial to assess whether the transfer of ion parameters to a different water model yields physically meaningful results. The aim of this work, is to determine parameters for $\mathrm{Mg}^{2+}$ in OPC water that leverage the strengths of both force fields, reproduce a broad range of experimental properties and lead to accurate simulation results of biomolecular systems.

Water constitutes the major part in simulations of membranes, proteins, and nucleic acids. Due to its distinct role, a large variety of water models exists which differ in their complexity, accuracy, and computational efficiency ${ }^{4,5}$. One of the most promising recent water models is the 4-site OPC mode ${ }^{5,6}$. OPC water was developed to accurately reproduce the electrostatic properties of water. It is quoted to improve simulations of intrinsically disordered proteins ${ }^{7,8}$. Balancing the interactions between the water molecules and amino acids is particularly important for disordered proteins since most models tend to favor overly compact and collapsed structures 7,9 . Tian et al. recommend to employ OPC water in combination with their recently developed protein force field ff $19 \mathrm{SB}^{10}$. In addition, the description of hydration of small molecules $^{6}$ as well as the thermodynamics of ligand binding ${ }^{11}$ improves with OPC water. Moreover, small RNA fragments ${ }^{12}$ or central properties of DNA ${ }^{13,14}$ show significant improve- ment when simulated in OPC water.

The apparent success of OPC water in simulating biological system raises the immediate question which ion force field should be used to obtain reliable results. In particular, accurate parameters for $\mathrm{Mg}^{2+}$ are essential since these ions play a vital role in a large variety of physiological processes such as ATP hydrolysis ${ }^{15}$, cellular signaling ${ }^{16,17}$, or the catalytic activity of ribozymes ${ }^{18-20}$. Due to the prominent role of $\mathrm{Mg}^{2+}$, a variety of parameters for different water models exists today ${ }^{1,21-33}$. However, the development of accurate parameters for $\mathrm{Mg}^{2+}$ turned out to be notoriously difficult leading to various shortcomings of the parameters: (i) Simultaneously capturing the solvation free energy and the structure of the first hydration shell failed unless polarization effects were included explicitly 28,34 . (ii) Without further optimization, the existing parameters led to unrealistically slow exchange in the first hydration shell of $\mathrm{Mg}^{2+}$ rendering the simulation of ion binding events impossible ${ }^{35}$. (iii) The binding affinities to ion binding sites on biomolecules were typically overrated and needed further optimization ${ }^{1,31,33,36}$.

Recently, progress was made in tackling this problems by using a larger Lennard-Jones (LJ) parameter space and by modifying the standard combination rules ${ }^{1,33}$. In most biomolecular simulations, the pair potential between atoms $i$ and $j$ is modeled as sum of the Coulomb term and the 12-6 Lennard-Jones (LJ) potential,

$$
V\left(r_{\mathrm{ij}}\right)=\frac{q_{\mathrm{i}} q_{\mathrm{j}}}{4 \pi \varepsilon_{0} r_{\mathrm{ij}}}+4 \varepsilon_{\mathrm{ij}}\left[\left(\frac{\sigma_{\mathrm{ij}}}{r_{\mathrm{ij}}}\right)^{12}-\left(\frac{\sigma_{\mathrm{ij}}}{r_{\mathrm{ij}}}\right)^{6}\right],
$$

where $q_{\mathrm{i}}$ is the charge, $r_{\mathrm{ij}}$ the distance, and $\varepsilon_{0}$ the dielectric constant of vacuum. The LJ term contains the interaction strength $\varepsilon_{\mathrm{ij}}$ and the diameter $\sigma_{\mathrm{ij}}$. Increasing the $\mathrm{LJ}$ parameter space, renders the interaction between water and $\mathrm{Mg}^{2+}$ more attractive. This in turn allowed us to implicitly include polar- 
ization effect and to simultaneously reproduce the solvation free energy and the structure of the first hydration shell for 6 different water models ${ }^{1,33}$.

In addition, polarization effects provoke shortcomings of the standard combination rules for describing ion-ion interactions or the interaction of ions and biomolecules. By using scaling factors in the Lorentz-Berthelot combination rules $^{37-40}$, the deviations from the standard combination rule can be taken into account

$$
\sigma_{\mathrm{MgX}}=\lambda_{\sigma}^{\mathrm{X}} \cdot \frac{\sigma_{\mathrm{Mg}}+\sigma_{\mathrm{X}}}{2} ; \quad \varepsilon_{\mathrm{MgX}}=\lambda_{\varepsilon}^{\mathrm{X}} \cdot \sqrt{\varepsilon_{\mathrm{Mg}} \varepsilon_{\mathrm{X}}}
$$

In the following, we will focus on $\mathrm{MgCl}_{2}$ solutions solutions and the binding of $\mathrm{Mg}^{2+}$ to RNA. Hence, $\mathrm{X}$ represents the $\mathrm{Cl}^{-}$anion or the atoms of an RNA. The scaling parameters $\lambda_{\sigma}$ and $\lambda_{\varepsilon}$ take some of the effects of polarizability into account thereby reproducing the activity derivative of $\mathrm{MgCl}_{2}$ solutions and correcting the excessive binding of the cations ${ }^{1,33}$.

In the following, we systematically evaluate the transferability of $\mathrm{Mg}^{2+}$ force field parameters, that were optimized previously as described above for different water models, to OPC water. Our results show that two parameter sets, called microMg and nanoMg, that were initially optimized in combination with SPC/E water, perform best in reproducing a broad variety of experimental properties.

\section{METHODS}

In the following, we use the 12 different $\mathrm{Mg}^{2+}$ parameters sets 1,33 that were optimized in combination with TIP3P ${ }^{41}$, SPC/E ${ }^{42}$, TIP3P-fb ${ }^{43}$, TIP4P/2005 ${ }^{44}$, TIP4P-Ew ${ }^{45}$, and TIP4P-D ${ }^{9}$ water.

The parameters of the water models are listed in Section S1 of the SI and the $\mathrm{Mg}^{2+}$ force field parameters are available from github https://github.com/bio-phys/ Magnesium-FFs and https://github.com/bio-phys/ optimizedMgFFs or from Section S2. Note that for each water model two parameter sets, labeled microMg and nanoMg, exist. For all the different water models, the micro $M g$ parameter set reproduces the experimental water exchange rate on the microsecond timescale while the nanoMg parameter set yields accelerated exchanges on the nanosecond timescale. In addition, we use the 12-6 and the 12-6-4 Li-Merz ${ }^{32}$ parameters that were optimized in combination with OPC water.

All simulations were done with GROMACS ${ }^{46}$ (version 2020) with the exception of the 12-6-4 parameters which use an additional term to incorporate polarization effects. The simulations with the 12-6-4 parameters were done with $\mathrm{AMBER}^{47}$ (version 2018). For more details on the simulations see Section S3 of the SI.

The simulations to evaluate the transferability of different force field parameters to OPC water were done in three consecutive steps:

(i) The single-ion properties were obtained for the 12 parameter sets for $\mathrm{Mg}^{2+}$ and the corresponding $\mathrm{Cl}^{-}$parameters (Mamatkulov-Schwierz ${ }^{29}$ for TIP3P, Smith-Deng ${ }^{48}$ for SPC/E, Grotz-Schwierz ${ }^{1}$ for TIP3P-fb, TIP4P/2005, TIP4PEw, and TIP4P-D). Values for the Li-Merz parameters (both
TABLE I. Optimal force field parameters for simulations of $\mathrm{Mg}^{2+}$ in OPC water. The parameters were previously optimized for SPC/E ${ }^{1}$ and reproduce the broad range of experimental properties when transferred to OPC (Table II). $\sigma_{\mathrm{ii}}, \varepsilon_{\mathrm{ii}}, \sigma_{\mathrm{io}}, \varepsilon_{\mathrm{io}}$ are the ion-ion and ion-water LJ parameters. $\lambda_{\sigma}^{\mathrm{X}}$ and $\lambda_{\varepsilon}^{\mathrm{X}}$ are the scaling factors for the Lorentz-Berthelot combination rules (eq 2) for the interaction with $\mathrm{Cl}^{-}$or the RNA atoms. Note that the scaling factors are only valid in combination with the $\mathrm{Cl}^{-}$parameters from Smith-Dang ${ }^{48}$ for SPC/E water, and the parmBSC $0 \chi_{\mathrm{OL} 3}$ RNA parameters ${ }^{49-51}$.

\begin{tabular}{lc||cc}
\multicolumn{1}{c||}{} & & microMg & nanoMg \\
\hline \hline$\sigma_{\text {ii }}$ & {$[\mathrm{nm}]$} & 0.1036 & 0.1046 \\
$\varepsilon_{\mathrm{ii}}$ & {$[\mathrm{kJ} / \mathrm{mol}]$} & 290.58 & 470.70 \\
$\sigma_{\mathrm{io}}$ & {$[\mathrm{nm}]$} & 0.2101 & 0.2106 \\
$\varepsilon_{\mathrm{io}}$ & {$[\mathrm{kJ} / \mathrm{mol}]$} & 16.08 & 20.47 \\
\hline$\lambda_{\sigma}^{\mathrm{Cl}}$ & & 1.59 & 1.59 \\
$\lambda_{\varepsilon}^{\mathrm{Cl}}$ & & 0.10 & 0.10 \\
$\sigma_{\mathrm{MgCl}}$ & {$[\mathrm{nm}]$} & 0.4316 & 0.4325 \\
$\varepsilon_{\mathrm{MgCl}}$ & {$[\mathrm{kJ} / \mathrm{mol}]$} & 1.0989 & 1.3986 \\
\hline$\lambda_{\sigma}^{\mathrm{RNA}}$ & & 1.1019 & 1.1107 \\
$\lambda_{\varepsilon}^{\mathrm{RNA}}$ & & 0.4856 & 0.3300 \\
$\sigma_{\mathrm{MgOP}}$ & {$[\mathrm{nm}]$} & 0.2202 & 0.2225 \\
$\varepsilon_{\mathrm{MgOP}}$ & {$[\mathrm{kJ} / \mathrm{mol}]$} & 7.7589 & 6.7111 \\
\hline \multicolumn{2}{l}{}
\end{tabular}

12-6 and 12-6-4) optimized for OPC were taken from their original publication ${ }^{32}$. As corresponding $\mathrm{Cl}^{-}$parameters, the Sengupta-Merz ${ }^{52}$ parameters optimized for OPC (both 12-6 and 12-6-4) were considered.

We calculated the solvation free energy of neutral $\mathrm{MgCl}_{2}$ ion pairs which includes the enthalpic and entropic contribution of the ions in water. In the computation, we took correction terms for finite size $e^{53}$, pressure ${ }^{54}$, and surface effects ${ }^{54}$ into account. In addition, we considered the radius and coordination number as the most important structural parameters to characterize the first hydration shell. More details on the simulations can be found in Section S4 of the SI.

(ii) For the 8 parameter sets that performed best in the previous step, we computed water exchange rates in the first hydration shell. The exchange rate was obtained by counting transitions of water molecules between the first and second hydration shell in long $\mathrm{MgCl}_{2}$ trajectories. More details on these computations can be found in Section S5 of the SI.

(iii) Finally, the activity derivative in $\mathrm{MgCl}_{2}$ and the binding affinity toward one of the non-bridging phosphate oxygens of a dimethylphosphate (DMP) were calculated, see also Section $\mathrm{S} 6$ and $\mathrm{S} 7$ of the SI. For DMP, the GAFF ${ }^{55}$ and AMBER RNA force field parameters (parmBSC $0 \chi_{\mathrm{OL} 3}{ }^{49-51}$ ) were used (see ref. ${ }^{33}$ for more details on the parametrization). Note that for the accuracy of these results the scaling factors, $\lambda_{\varepsilon}^{\mathrm{Cl}}, \lambda_{\sigma}^{\mathrm{Cl}}$, $\lambda_{\varepsilon}^{\mathrm{RNA}}$, and $\lambda_{\sigma}^{\mathrm{RNA}}$ according to eq 2 are crucial. The scaling factors for the different water models are given in Section S2.

As consistency check, the binding affinities for the final two parameter sets were calculated from two different methods, namely by integrating potentials of mean force and via alchemical transformations. More details on the calculations can be found in Section S7 of the SI. 
TABLE II. Results for single-ion, ion-ion and ion-RNA properties in OPC for the optimal $\mathrm{Mg}^{2+}$ parameters transferred from SPC/E water (Table I). Solvation free energy of neutral $\mathrm{MgCl}_{2}$ ion pairs $\Delta G_{\text {solv }}, \mathrm{Mg}^{2+}$-oxygen distance in the first hydration shell $R_{1}$, coordination number of the first hydration shell $n_{1}$, water exchange rate from the first hydration shell $k$, binding affinity toward the phosphate oxygen of DMP $\Delta G_{\mathrm{b}}^{0}$, $\mathrm{Mg}^{2+}$-phosphate oxygen distance in inner-sphere coordination $R_{\mathrm{b}}$, and $a_{c c}$ the activity derivative of a $\mathrm{MgCl}_{2}$ solution at $0.25 \mathrm{M}$ concentration. The experimental value for $\Delta G_{\mathrm{b}}^{0}$ is derived from the $\log$ stability constant $(\log K=0.45)$ given in ref. ${ }^{56}$.

\begin{tabular}{|c|c|c|c|c|}
\hline & & microMg & nanoMg & exp. \\
\hline$\Delta G_{\text {solv }}$ & {$[\mathrm{kJ} / \mathrm{mol}]$} & $-2628.7 \pm 1$ & $-2535.9 \pm 1$ & $-2532^{57}$ \\
\hline$R_{1}$ & {$[\mathrm{~nm}]$} & $0.212 \pm 0.004$ & $0.215 \pm 0.004$ & $0.209 \pm 0.004^{58}$ \\
\hline$n_{1}$ & & 6 & 6 & $6^{58}$ \\
\hline$k$ & {$\left[\mathrm{~s}^{-1}\right]$} & $(1.22 \pm 0.5) \cdot 10^{5}$ & $(2.86 \pm 0.07) \cdot 10^{7}$ & $(5.3-6.7) \times 10^{559,60}$ \\
\hline$a_{c c}$ & & $0.88 \pm 0.01$ & $0.88 \pm 0.03$ & $0.93^{61}$ \\
\hline$\Delta G_{\mathrm{b}}^{0}$ & {$\left[\mathrm{k}_{\mathrm{B}} \mathrm{T}\right]$} & $-0.818 \pm 0.487$ & $-0.969 \pm 0.298$ & $-1.036^{56}$ \\
\hline$R_{\mathrm{b}}$ & {$[\mathrm{nm}]$} & $0.207 \pm 0.004$ & $0.207 \pm 0.004$ & $0.206-0.208^{62}$ \\
\hline
\end{tabular}

\section{RESULTS}

The aim of this work, is to evaluate the transferability of different $\mathrm{Mg}^{2+}$ force fields to OPC water. In particular, we calculate all physical properties that were targeted in the initial optimization including the solvation free energy, the distance to water oxygens in the first hydration shell, the hydration number, the activity coefficient derivative in $\mathrm{MgCl}_{2}$ solutions, and the binding affinity and distance to the phosphate oxygens on nucleic acids. Finally, we select two parameter sets (Table I), micro $M g$ and nano $M g$, that reproduce the broad range of experimental properties (Table II) and lead to accurate simulation results in OPC water and are recommended for conventional or enhanced sampling purposes, respectively.

\section{A. Solvation free energy, $\mathrm{Mg}^{2+}$-water distance, and coordination number}

To evaluate whether the $\mathrm{Mg}^{2+}$ parameters, that were optimized in combination with different 3- and 4-site water models, are transferable, we calculated the solvation free energy of $\mathrm{MgCl}_{2}$ ion pairs in OPC water. Note that the usage of neutral ion pairs is more robust since it does not rely on conflicting experimental results for the hydration free energy of a proton $^{57,63,64}$. Figure 1A shows the deviation of the calculated solvation free energy from the experimental results $\Delta \Delta G_{\text {solv }}$ for the transferred parameters. For comparison, the values for the OPC-optimized 12-6 and 12-6-4 Li-Merz parameters ${ }^{32}$ are shown. The simulations with the parameters transferred from SPC/E to OPC water yield the smallest deviation from experiments and perform even slightly better than those optimized directly within OPC.

Interestingly, $\Delta \Delta G_{\text {solv }}$ is smaller for the parameter transferred from 3-site waters (SPC/E, followed by TIP3P-fb and TIP3P). 4-site water models (TIP4P-D, followed by TIP4PEw, and TIP4P/2005) lead to larger deviations despite the fact that OPC is also a 4-site model (Table S4). This result has not been expected based on previous work which showed reasonable transferability of ion parameters within water models of the same complexity ${ }^{1,2,65}$.

The size of the first hydration shell, measured by the dis- tance between $\mathrm{Mg}^{2+}$ and the water oxygens $R_{1}$, agrees well with the experimental results for all parameter sets transferred to OPC water (Figure 1B, Table S4). On the other hand, the OPC-optimized 12-6 model by Li-Merz significantly underestimates $R_{1}$. This shortcoming led to the development of the OPC-optimized 12-6-4 model which provides much better agreement (Figure 1B).

For all parameter sets, the coordination number in OPC water is 6 and precisely matches the experimental result (Table $\mathrm{S} 4)$.

Similarly, the simulations with $\mathrm{Cl}^{-}$parameter from different water models transferred to OPC closely reproduce $R_{1}$ (Figure 1C, Table S5). On the other hand, the OPC-optimized 12-6 Sengupta-Merz parameters deviate noticeably from the experimental value while the 12-6-4 version yields good agreement.

In summary, the six $\mathrm{Mg}^{2+}$ parameter sets that were previously optimized for the 3-site water models (SPC/E, followed by TIP3P-fb and TIP3P) and the corresponding three $\mathrm{Cl}^{-}$parameter sets yield the best results for the solvation free energy and structure of the first hydration shell when transferred to OPC water and are used in the subsequent steps.

\section{B. Water exchange rate}

Including water exchange rates in the evaluation of the transferability is important to correctly capture ion binding and to avoid shortcomings due to unrealistically slow exchange dynamics as observed previously ${ }^{35}$. In particular, we evaluated the capability of micro $M g$ in reproducing the experimental exchange rate and the capability of nanoMg to speeding up the association or dissociation processes for application in enhanced sampling simulations. Figure 2 shows the water exchange rate for the six parameter sets transferred to OPC water in comparison to the OPC-optimized Li-Merz parameters and the experimental results ${ }^{59,60}$. All transferred parameter sets yield a lower exchange rate in OPC water compared to the original water reflecting the influence of the water model on the exchange kinetics ${ }^{3}$. Still, the TIP3P-fb and SPC/E-optimized microMg parameters yield the same order of magnitude as in experiments and are therefore considered reasonably accurate (Table S6). In addition, the transferred microMg parameters perform significantly better compared to 

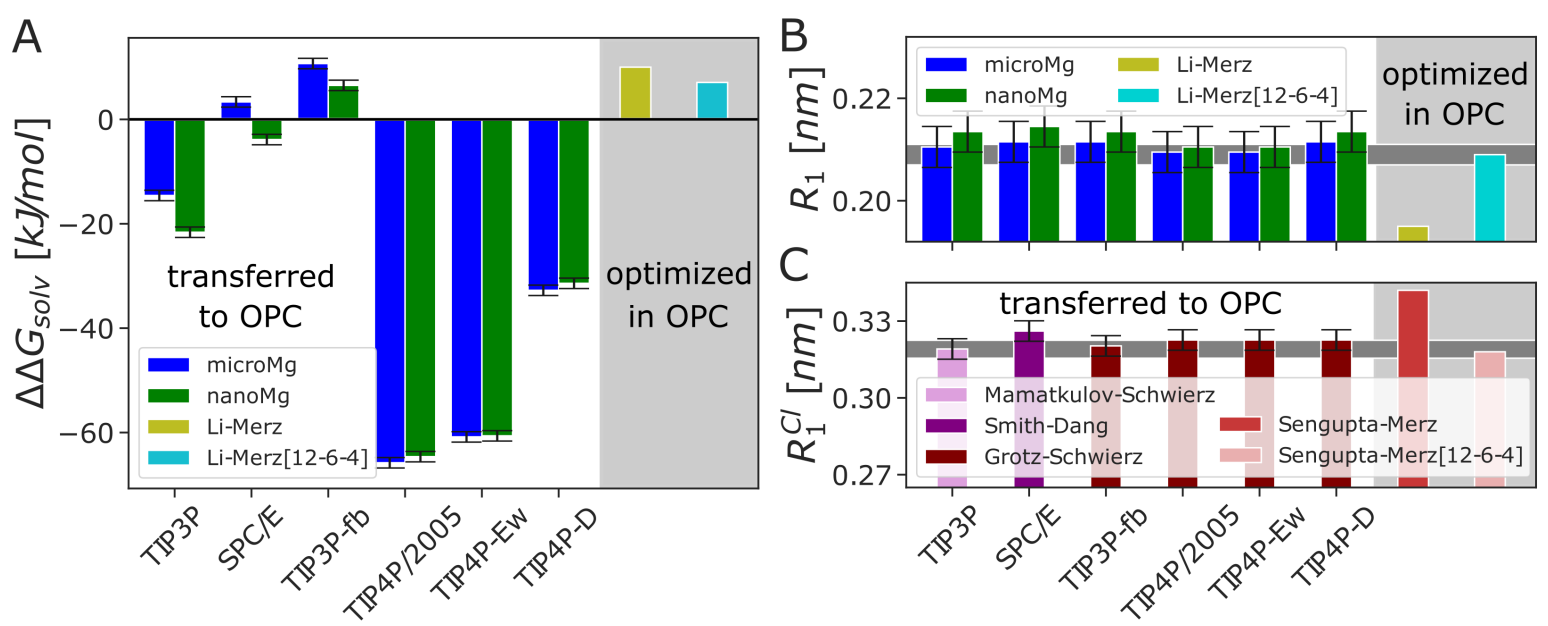

FIG. 1. (A) Deviation of the solvation free energy from the experimental results ${ }^{57} \Delta \Delta G_{\text {solv }}$ for neutral $\mathrm{MgCl}_{2}$ ion pairs. The results are obtained by transferring the $\mathrm{Mg}^{2+}$ parameters obtained in the different water models (TIP3P, SPC/E, TIP3P-fb, TIP4P/2005, TIP4P-Ew, or TIP4P-D) to OPC. For comparison, the OPC-optimized $12-6$ and 12-6-4 Li-Merz ${ }^{32}$ parameters are shown. Since ref. ${ }^{32}$ only contains values for $\mathrm{Mg}^{2+}$, the experimental value for $\mathrm{Cl}^{-57}$ was added to obtain a neutral ion pair. (B) $\mathrm{Mg}^{2+}$ - oxygen distance $R_{1}$ of the first hydration shell. (C) $\mathrm{Cl}^{-}$- oxygen distance $R_{1}^{\mathrm{Cl}}$ of the first hydration shell obtained with different force fields from the literature ${ }^{1,29,48,52}$. The gray horizontal bar in $(\mathrm{B}, \mathrm{C})$ corresponds to the experimental value ${ }^{58}$.

the 12-6 and 12-6-4 Li-Merz parameters. Note, however, that there is a large uncertainty in the rate for the 12-6 Li-Merz parameters as the exchange dynamics is very slow with only 12 exchanges in $2 \mu \mathrm{s}$. With the 12-6-4 parameters, the exchange is even slower and not a single exchange event could be observed (Table S6).

For the nanoMg parameter sets, significant differences are observed: With the SPC/E-optimized parameters in OPC, exchanges happen on the order of $10^{7} / \mathrm{s}$ and are therefore almost as fast as in SPC/E $\left(10^{8} / \mathrm{s}\right)^{1}$. With the TIP3P-fb-optimized and TIP3P-optimized nanoMg parameters transferred to OPC, the maximum rate is on the same order of magnitude $\left(10^{6} \% \mathrm{~s}\right)$ and two orders of magnitude slower $\left(10^{8} / \mathrm{s}\right.$ to $\left.10^{6} \% \mathrm{~s}\right)$, respectively. The SPC/E-optimized nanoMg parameters in OPC are therefore most beneficial for enhanced sampling of ion binding in OPC water.

In summary, based on the water exchange rate, the SPC/Eand TIP3P-fb-optimized parameters yield the best agreement with experimental results (microMg sets) while the $\mathrm{SPC} / \mathrm{E}-$ optimized nanoMg yield the highest acceleration of exchanges.

\section{Activity derivative and binding affinity to RNA}

Finally, we evaluate the transferability based on the activity derivative and the binding affinity and distance to the phosphate oxygens on nucleic acids. The activity derivative $a_{\mathrm{cc}}$ is important in the evaluation of the transferability since it gives information on the balance between ion-ion and ion-water interactions ${ }^{37}$. Figure $3 \mathrm{~A}$ shows that the TIP3P-, SPC/E- and TIP3P-fb-optimized parameters reproduce $a_{\mathrm{cc}}$ over a broad concentration range in OPC water.
The binding affinity $\Delta G_{\mathrm{b}}^{0}$ and distance $R_{\mathrm{b}}$ provide insight into the accuracy of the $\mathrm{Mg}^{2+}$-RNA interactions and are shown in Figure 3B. The SPC/E-optimized parameters precisely match $\Delta G_{\mathrm{b}}^{0}$ and $R_{\mathrm{b}}$ in OPC water, whereas parameters for TIP3P overestimate and those for TIP3P-fb underestimate $\Delta G_{\mathrm{b}}^{0}$.

In summary, the SPC/E-optimized microMg and nanoMg parameters yield the best agreement with the experimental results in OPC water.

Figure $3 \mathrm{C}$ provides additional insight into the ion binding process from the free energy profiles along the $\mathrm{Mg}^{2+}$ phosphate oxygen distance for the SPC/E-optimized parameters in OPC. The two minima correspond to the inner- and outer-sphere coordination of $\mathrm{Mg}^{2+}$ and are identical for $m i$ croMg and nanoMg, as expected. The energetic barriers, on the other hand, is significantly lower for nanoMg reflecting its enhanced ion binding kinetics.

\section{CONCLUSION}

OPC water has proven to be one of the most promising 4site water models ${ }^{6}$ due to its improved description of small molecules $^{6,11}$, proteins $s^{7,8,10}$ and nucleic acids ${ }^{12-14}$. In this work, we evaluate the transferability of twelve different $\mathrm{Mg}^{2+}$ force fields to OPC water based on solvation free energy, size of the first hydration shell, hydration number, water exchange rate, activity derivative, and binding affinity to the phosphate oxygens on nucleic acids. Our results show that the force field parameters, that were previously optimized with $\mathrm{SPC} / \mathrm{E}^{1}$, are best suited to reproduce the broad range of solution properties in OPC water. In addition, the results show that the transferred parameters are compatible or better than OPC-optimized 12-6 


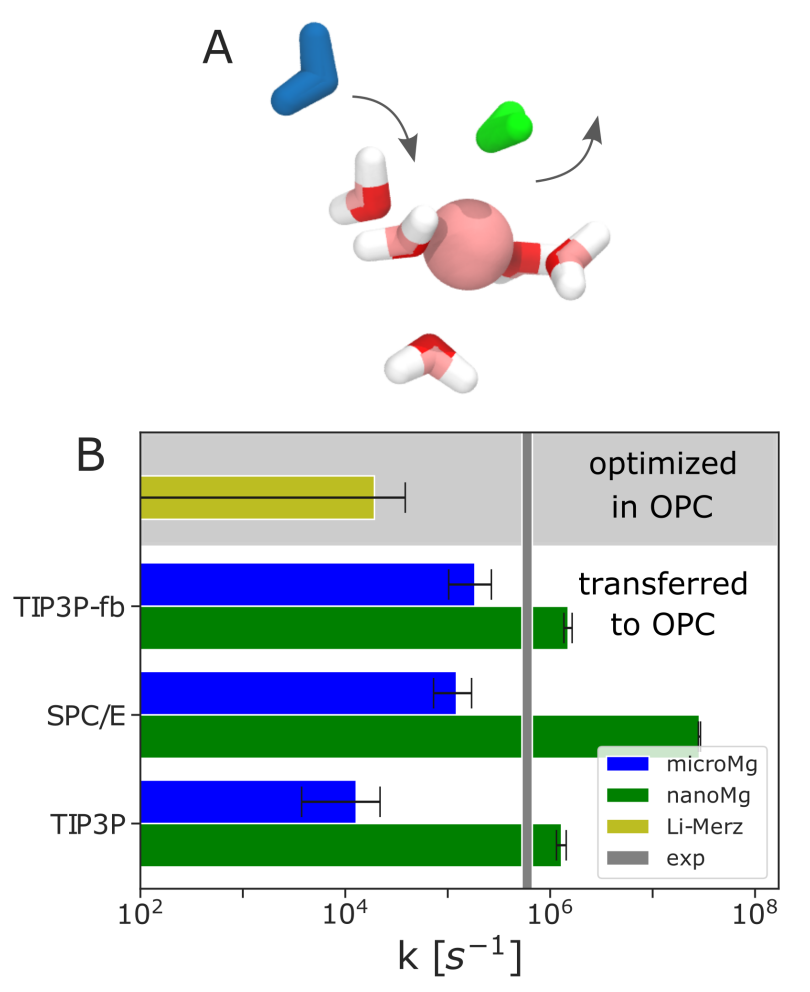

FIG. 2. (A) Snapshot of a $\mathrm{Mg}^{2+}$ ion including the first hydration shell during a direct water exchange: The incoming water is shown in blue, the outgoing water in green. (B) Water exchange rates calculated from $2 \mu \mathrm{s}$ of $1 \mathrm{M} \mathrm{MgCl}_{2}$ trajectories for the different force field parameters. The gray vertical bar indicates the experimental rate ${ }^{59,60}$. Errors are calculated from block averaging by dividing the trajectory into 2 blocks.

and 12-6-4 parameters ${ }^{32}$ in reproducing the experimental solvation free energy and water exchange rate.

Moreover, the SPC/E-optimized parameters closely reproduce the binding affinity of $\mathrm{Mg}^{2+}$ toward the phosphate oxygen of RNA. Matching the binding affinity of metal cations to specific ion binding sites is particularly important since it improves the agreement between experiments and simulations for the structure and properties of larger nucleic acid systems ${ }^{66}$.

In summary, the SPC/E-optimized parameter sets microMg and nanoMg provide an efficient and highly accurate model for the simulation of $\mathrm{Mg}^{2+}$ in OPC water.

We recommend to use microMg to obtain accurate water exchange kinetics comparable to experimental results on the microsecond timescale. Further, we recommend to use the nanoMg parameters to yield accelerated exchange kinetics and ion-binding in enhanced sampling setups.

\section{ACKNOWLEDGEMENTS}

We acknowledge financial support from the DFG (Emmy Noether program, Grant No. 315221747 and the CRC902). GOETHE HLR is acknowledged for supercomputing access.
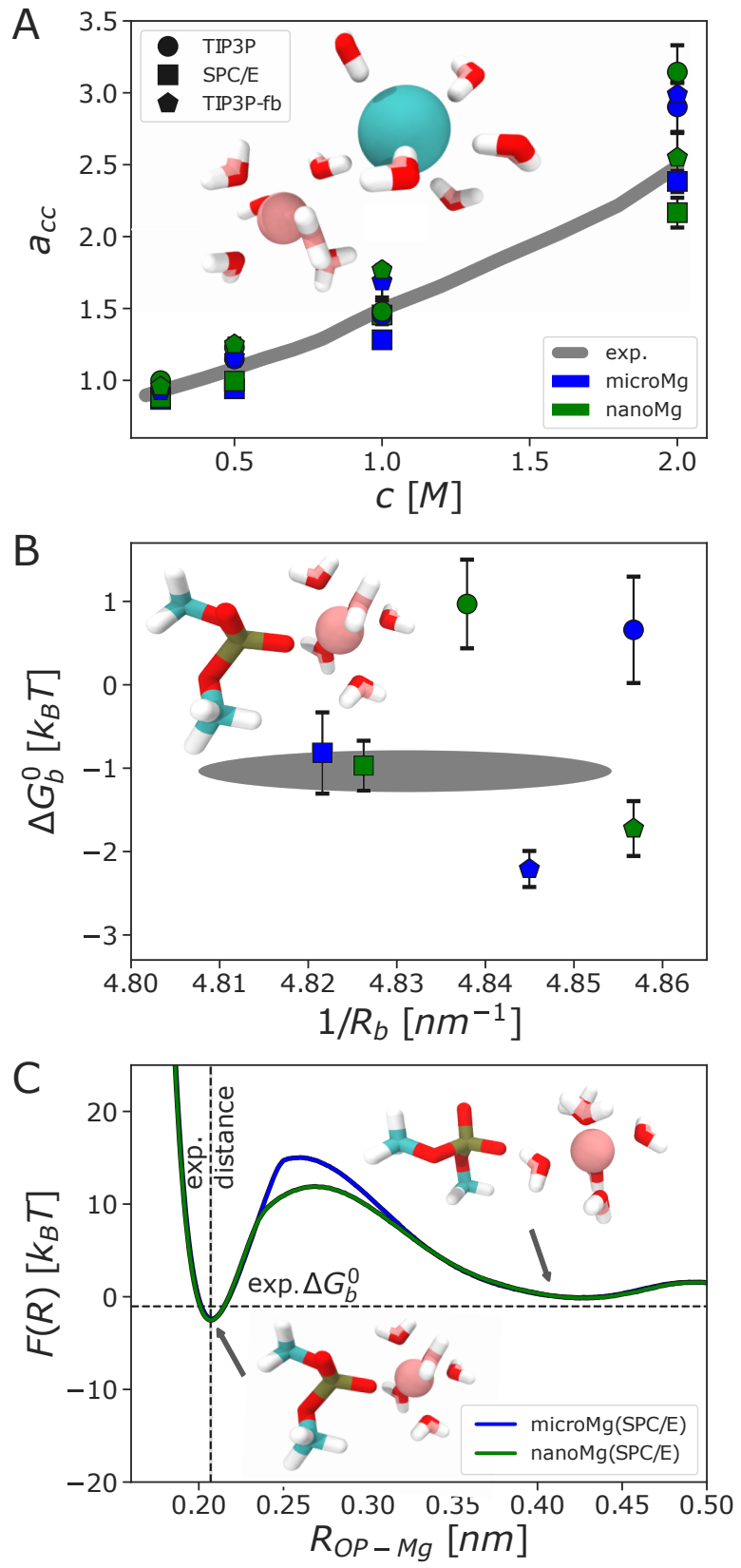

FIG. 3. (A) Activity derivative $a_{\mathrm{cc}}$ as function of the $\mathrm{MgCl}_{2}$ concentration for selected force field parameters. The inset shows a $\mathrm{Mg}^{2+}$ $\mathrm{Cl}^{-}$pair and their first hydration shells. The gray line corresponds to the experimental value from ref. ${ }^{61}$. (B) Binding affinity $\Delta G_{\mathrm{b}}^{0}$ in correlation with the inverse of the binding distance $1 / R_{\mathrm{b}}$ toward the phosphate oxygen. The inset shows a simulation snapshot of the DMP molecule with one $\mathrm{Mg}^{2+}$ ion in inner-sphere coordination. The gray area represents the experimental value from refs. ${ }^{56,62}$. (C) Free energy profiles $F(R)$ along the $\mathrm{Mg}^{2+}$-phosphate oxygen distance for the optimal parameters sets in OPC water. The insets show simulation snapshots of $\mathrm{Mg}^{2+}$ in the two stable states. 
bioRxiv preprint doi: https://doi.org/10.1101/2021.12.07.471562; this version posted December 7, 2021. The copyright holder for this preprint (which was not certified by peer review) is the author/funder, who has granted bioRxiv a license to display the preprint in perpetuity. It is made available under aCC-BY 4.0 International license.

\section{SUPPORTING INFORMATION}

Further details of the simulations and the analysis are provided in the supplementary material: Simulation setup, parameters of different water models and $\mathrm{Mg}^{2+}$ force fields, details on the computation of the solvation free energy, water exchange rates, activity derivatives, and binding affinity. The $\mathrm{Mg}^{2+}$ force field parameters are also available at https://github.com/bio-phys/MgFF_OPC.

\section{DATA AVAILABILITY}

The data that support the finding of this study are available from the corresponding author upon reasonable request.

${ }^{1}$ K. K. Grotz and N. Schwierz, "Optimized Magnesium Force Field Parameters for Biomolecular Simulations with Accurate Solvation, Ion-Binding, and Water-Exchange Properties in SPC/E, TIP3P-fb, TIP4P/2005, TIP4PEw, and TIP4P-D," J. Chem. Theory Comput. , accepted.

${ }^{2}$ M. F. Döpke, O. A. Moultos, and R. Hartkamp, "On the transferability of ion parameters to the TIP4P/2005 water model using molecular dynamics simulations," J. Chem. Phys. 152, 024501 (2020).

${ }^{3}$ S. Falkner and N. Schwierz, "Kinetic pathways of water exchange in the first hydration shell of magnesium: Influence of water model and ionic force field," J Chem. Phys. 155 (2021), 10.1063/5.0060896.

${ }^{4}$ C. Vega and J. L. F. Abascal, "Simulating water with rigid non-polarizable models: a general perspective," Phys. Chem. Chem. Phys. 13, 19663-19688 (2011).

${ }^{5}$ S. P. K. Pathirannahalage, N. Meftahi, A. Elbourne, A. C. G. Weiss, C. F. McConville, A. Padua, D. A. Winkler, M. Costa Gomes, T. L. Greaves, Q. A. Besford, T. C. Le, and A. J. Christofferson, "A systematic comparison of the structural and dynamic properties of commonly used water models for molecular dynamics simulations,' J. Chem. Inf. Model 61, 4521-4536 (2021).

${ }^{6} \mathrm{~S}$. Izadi, R. Anandakrishnan, and A. V. Onufriev, "Building water models: A different approach,” J. Phys. Chem. Lett. 5, 3863-3871 (2014), arXiv: 1408.1679 .

${ }^{7}$ P. S. Shabane, S. Izadi, and A. V. Onufriev, "General Purpose Water Model Can Improve Atomistic Simulations of Intrinsically Disordered Proteins," J. Chem. Theory Comput. 15, 2620-2634 (2019).

${ }^{8}$ D. Pantoja-Uceda, J. L. Neira, L. M. Contreras, C. A. Manton, D. R. Welch, and B. Rizzuti, "The isolated C-terminal nuclear localization sequence of the breast cancer metastasis suppressor 1 is disordered," Arch. Biochem. Biophys. 664, 95-101 (2019).

${ }^{9}$ S. Piana, A. G. Donchev, P. Robustelli, and D. E. Shaw, "Water dispersion interactions strongly influence simulated structural properties of disordered protein states," J. Phys. Chem. B 119, 5113-5123 (2015).

${ }^{10}$ C. Tian, K. Kasavajhala, K. A. Belfon, L. Raguette, H. Huang, A. N. Migues, J. Bickel, Y. Wang, J. Pincay, Q. Wu, and C. Simmerling, "Ff19SB: Amino-Acid-Specific Protein Backbone Parameters Trained against Quantum Mechanics Energy Surfaces in Solution," J. Chem. Theory Comput. 16, 528-552 (2020).

${ }^{11}$ K. Gao, J. Yin, N. M. Henriksen, A. T. Fenley, and M. K. Gilson, "Binding Enthalpy Calculations for a Neutral Host-Guest Pair Yield Widely Divergent Salt Effects across Water Models," J. Chem. Theory Comput. 11, 4555-4564 (2015).

${ }^{12}$ C. Bergonzo and T. E. Cheatham, "Improved Force Field Parameters Lead to a Better Description of RNA Structure," J. Chem. Theory Comput. 11, 3969-3972 (2015).

${ }^{13} \mathrm{~F}$. Häse and M. Zacharias, "Free energy analysis and mechanism of base pair stacking in nicked DNA," Nucleic Acids Res. 44, 7100-7108 (2016).

${ }^{14}$ R. Galindo-Murillo, J. C. Robertson, M. Zgarbová, J. Šponer, M. Otyepka, P. Jurečka, and T. E. Cheatham, "Assessing the Current State of Amber Force Field Modifications for DNA," J. Chem. Theory Comput. 12, 41144127 (2016)
${ }^{15}$ N. H. Williams, "Magnesium Ion Catalyzed ATP Hydrolysis," J. Am. Chem. Soc. 122, 12023-12024 (2000).

${ }^{16}$ J. H. de Baaij, J. G. Hoenderop, and R. J. Bindels, "Magnesium in man: Implications for health and disease," Physiol. Rev. 95, 1-46 (2015).

${ }^{17}$ A. Stangherlin and J. S. O'Neill, "Signal Transduction: Magnesium Manifests as a Second Messenger," Curr. Biology 28, R1403-R1405 (2018).

${ }^{18} \mathrm{~J}$. A. Cowan, "Structural and catalytic chemistry of magnesium-dependent enzymes," Biometals 15, 225-235 (2002).

${ }^{19}$ A. Pyle, "Metal ions in the structure and function of RNA," J. Biol. Inorg. Chem. 7, 679-690 (2002)

${ }^{20}$ R. K. O. Sigel and A. M. Pyle, "Alternative Roles for Metal Ions in Enzyme Catalysis and the Implications for Ribozyme Chemistry," Chem. Rev. 107, 97-113 (2007).

${ }^{21}$ J. Åqvist, "Ion-water interaction potentials derived from free energy perturbation simulations," J. Phys. Chem. 94, 8021-8024 (1990).

${ }^{22}$ C. S. Babu and C. Lim, "Empirical force fields for biologically active divalent metal cations in water," J. Phys. Chem. A 110, 691-699 (2006).

${ }^{23}$ D. M. Mayaan, Evelyn; Moser, Adam; MacKerell, Aalexander D. Jr., York, "CHARMM Force Field Parameters for Simulation of Reactive Intermediates in Native and Thio-Substituted Ribozymes," J. Comput. Chem. 28, 495-507 (2007)

${ }^{24}$ E. Duboué-Dijon, P. Delcroix, H. Martinez-Seara, J. Hladílková, P. Coufal, T. Křrižek, and P. Jungwirth, "Binding of Divalent Cations to Insulin: Capillary Electrophoresis and Molecular Simulations," J. Phys. Chem. B 122, 5640-5648 (2018).

${ }^{25}$ O. Allnér, L. Nilsson, and A. Villa, "Magnesium Ion-Water Coordination and Exchange in Biomolecular Simulations," J. Chem. Theory Comput. 8, 1493-1502 (2012).

${ }^{26}$ S. Mamatkulov, M. Fyta, and R. R. Netz, "Force fields for divalent cations based on single-ion and ion-pair properties," J. Chem. Phys. 138, 24505 (2013).

${ }^{27} \mathrm{P}$. Li, B. P. Roberts, D. K. Chakravorty, and K. M. Merz, "Rational design of particle mesh ewald compatible lennard-jones parameters for +2 metal cations in explicit solvent," J. Chem. Theory Comput. 9, 2733-2748 (2013).

${ }^{28} \mathrm{P}$. Li and K. M. Merz, "Taking into Account the Ion-Induced Dipole Interaction in the Nonbonded Model of Ions," J. Chem. Theory Comput. 10, 289-297 (2014).

${ }^{29}$ S. Mamatkulov and N. Schwierz, "Force fields for monovalent and divalent metal cations in TIP3P water based on thermodynamic and kinetic properties," J. Chem. Phys. 148, 74504 (2018).

${ }^{30}$ H. T. Nguyen, N. Hori, and D. Thirumalai, "Theory and simulations for RNA folding in mixtures of monovalent and divalent cations," Proc. Natl. Acad. Sci. 116, 21022-21030 (2019).

${ }^{31}$ S. Cruz-León, K. K. Grotz, and N. Schwierz, "Extended magnesium and calcium force field parameters for accurate ion-nucleic acid interactions in biomolecular simulations," J. Chem. Phys. 154, 171102 (2021).

${ }^{32} \mathrm{Z}$. Li, L. F. Song, P. Li, and K. M. Merz, "Systematic Parametrization of Divalent Metal Ions for the OPC3, OPC, TIP3P-FB, and TIP4P-FB Water Models," J. Chem. Theory Comput. 16, 4429-4442 (2020).

${ }^{33}$ K. K. Grotz, S. Cruz-León, and N. Schwierz, "Optimized Magnesium Force Field Parameters for Biomolecular Simulations with Accurate Solvation, Ion-Binding, and Water-Exchange Properties," J. Chem. Theory Comput. 17, 2530-2540 (2021).

${ }^{34}$ I. M. Zeron, J. L. Abascal, and C. Vega, "A force field of $\mathrm{Li}^{+}, \mathrm{Na}^{+}, \mathrm{K}^{+}$, $\mathrm{Mg}^{2+}, \mathrm{Ca}^{2+}, \mathrm{Cl}^{-}$, and $\mathrm{SO}_{4}{ }^{2-}$ In aqueous solution based on the TIP4P/2005 water model a," J. Chem. Phys. 151 (2019), 10.1063/1.5121392.

${ }^{35} \mathrm{~N}$. Schwierz, "Kinetic pathways of water exchange in the first hydration shell of magnesium," J. Chem. Phys. 152, 224106 (2020).

${ }^{36}$ M. T. Panteva, G. M. Giambacsu, and D. M. York, "Force field for $\mathrm{Mg}^{2+}$, $\mathrm{Mn}^{2+}, \mathrm{Zn}^{2+}$, and $\mathrm{Cd}^{2+}$ ions that have balanced interactions with nucleic acids," J. Phys. Chem. B 119, 15460-15470 (2015).

${ }^{37}$ M. B. Gee, N. R. Cox, Y. Jiao, N. Bentenitis, S. Weerasinghe, and P. E. Smith, "A Kirkwood-Buff Derived Force Field for Aqueous Alkali Halides," J. Chem. Theory Comput. 7, 1369-1380 (2011).

${ }^{38}$ M. Fyta and R. R. Netz, "Ionic force field optimization based on single-ion and ion-pair solvation properties: Going beyond standard mixing rules," J. Chem. Phys. 136, 124103 (2012).

${ }^{39} \mathrm{~S}$. Weerasinghe and P. E. Smith, "A Kirkwood-Buff derived force field for sodium chloride in water," J. Chem. Phys. 119, 11342-11349 (2003). 
${ }^{40}$ B. Hess and N. F. A. van der Vegt, "Cation specific binding with protein surface charges,” Proc. Natl. Acad. Sci. 106, 13296-13300 (2009).

${ }^{41}$ W. L. Jorgensen, J. Chandrasekhar, J. D. Madura, R. W. Impey, and M. L. Klein, "Comparison of simple potential functions for simulating liquid water," J. Chem. Phys. 79, 926-935 (1983).

${ }^{42}$ H. J. Berendsen, J. R. Grigera, and T. P. Straatsma, "The missing term in effective pair potentials," J. Phys. Chem. 91, 6269-6271 (1987).

${ }^{43}$ L. P. Wang, T. J. Martinez, and V. S. Pande, "Building force fields: An automatic, systematic, and reproducible approach," J. Phys. Chem. Lett. 5, 1885-1891 (2014).

${ }^{44}$ J. L. Abascal and C. Vega, "A general purpose model for the condensed phases of water: TIP4P/2005," J. Chem. Phys. 123 (2005), 10.1063/1.2121687.

${ }^{45}$ H. W. Horn, W. C. Swope, J. W. Pitera, J. D. Madura, T. J. Dick, G. L. Hura, and T. Head-Gordon, "Development of an improved four-site water model for biomolecular simulations: TIP4P-Ew," J. Chem. Phys. 120, 9665-9678 (2004).

${ }^{46}$ M. J. Abraham, T. Murtola, R. Schulz, S. Páll, J. C. Smith, B. Hess, and E. Lindah, "Gromacs: High performance molecular simulations through multi-level parallelism from laptops to supercomputers," SoftwareX 1-2, 19-25 (2015).

${ }^{47}$ D. A. Case, K. Belfon, I. Y. Ben-Shalom, S. R. Brozell, D. S. Cerutti, T. E. I. Cheatham, V. W. D. Cruzeiro, T. Darden, R. E. Duke, G. Giambasu, M. K. Gilson, H. Gohlke, A. W. Goetz, R. Harris, P. A. Izadi, S. Izmailov, K. Kasavajhala, A. Kovalenko, R. Krasny, T. Kurtzman, T. S. Lee, S. LeGrand, P. Li, C. Lin, J. Liu, T. Luchko, R. Luo, V. Man, K. M. Merz, Y. Miao, O. Mikhailovskii, G. Monard, H. Nguyen, A. Onufriev, F. Pan, S. Pantano, R. Qi, D. R. Roe, A. Roitberg, C. Sagui, S. SchottVerdugo, J. Shen, C. L. Simmerling, N. R. Skrynnikov, J. Smith, J. Swails, R. C. Walker, J. Wang, L. Wilson, R. M. Wolf, X. Wu, Y. Xiong, Y. Xue, D. M. York, and P. A. Kollman, "Amber 2018," (2018).

${ }^{48}$ D. E. Smith and L. X. Dang, "Computer simulations of $\mathrm{NaCl}$ association in polarizable water,” J. Chem. Phys. 100, 3757-3766 (1994).

${ }^{49}$ A. Pérez, I. Marchán, D. Svozil, J. Sponer, T. E. Cheatham, C. A. Laughton, and M. Orozco, "Refinement of the AMBER force field for nucleic acids: Improving the description of $\alpha / \gamma$ conformers," Biophys. J. 92, 3817-3829 (2007).

${ }^{50}$ P. Banáš, D. Hollas, M. Zgarbová, P. Jurečka, M. Orozco, T. E. Cheatham, J. Šponer, and M. Otyepka, "Performance of molecular mechanics force fields for RNA simulations: Stability of UUCG and GNRA hairpins," J. Chem. Theory Comput. 6, 3836-3849 (2010).

${ }^{51}$ M. Zgarbová, M. Otyepka, J. Šponer, A. Mládek, P. Banáš, T. E. Cheatham, and P. Jurečka, "Refinement of the Cornell et al. Nucleic acids force field based on reference quantum chemical calculations of glycosidic torsion profiles," J. Chem. Theory Comput. 7, 2886-2902 (2011).

${ }^{52}$ A. Sengupta, Z. Li, L. F. Song, P. Li, and K. M. Merz, "Parameterization of Monovalent Ions for the OPC3, OPC, TIP3P-FB, and TIP4P-FB Water Models," J. Chem. Inf. Model 61, 869-880 (2021).

${ }^{53}$ G. Hummer, L. R. Pratt, and A. E. García, "Free Energy of Ionic Hydration," J. Phys. Chem. 100, 1206-1215 (1996).

${ }^{54}$ D. Horinek, S. I. Mamatkulov, and R. R. Netz, "Rational design of ion force fields based on thermodynamic solvation properties," J. Chem. Phys. 130, 124507 (2009).

${ }^{55}$ J. Wang, R. M. Wolf, J. W. Caldwell, P. A. Kollman, and D. A. Case, "Development and testing of a general Amber force field," J. Comput. Chem. 25, 1157-1174 (2004).

${ }^{56}$ R. K. Sigel and H. Sigel, "A stability concept for metal ion coordination to single-stranded nucleic acids and affinities of individual sites," Acc. Chem. Res. 43, 974-984 (2010).

${ }^{57}$ Y. Marcus, Ion Properties (Marcel Dekker, Inc., New York, Basel, 1997).

${ }^{58}$ Y. Marcus, "Ionic Radii in Aqueous Solutions," Chem. Rev. 88, 1475-1498 (1988).

${ }^{59}$ J. Neely and R. Connick, "Rate of Water Exchange from Hydrated Magnesium Ion,” J. Am. Chem. Soc. 92, 3476-3478 (1970).

${ }^{60}$ A. Bleuzen, P.-A. Pittet, L. Helm, and A. E. Merbach, "Water exchange on magnesium(II) in aqueous solution: a variable temperature and pressure ${ }^{17}$ O NMR study,” Magn. Reson. Chem. 35, 765-773 (1997).

${ }^{61}$ R. A. Robinson and R. H. Stokes, Electrolyte Solutions, 2nd ed. (Dover, New York, 2002).
${ }^{62}$ F. Leonarski, L. D'Ascenzo, and P. Auffinger, " $\mathrm{Mg}^{2+}$ ions: Do they bind to nucleobase nitrogens?" Nucleic Acids Res. 45, 987-1004 (2017).

${ }^{63}$ M. D. Tissandier, K. A. Cowen, W. Y. Feng, E. Gundlach, M. H. Cohen, A. D. Earhart, J. V. Coe, and T. R. Tuttle, "The proton's absolute aqueous enthalpy and Gibbs free energy of solvation from cluster-ion solvation data," J. Phys. Chem. A 102, 7787-7794 (1998).

${ }^{64}$ T. L. Beck, "The influence of water interfacial potentials on ion hydration in bulk water and near interfaces," Chem. Phys. Lett. 561-562, 1-13 (2013).

${ }^{65}$ P. Loche, P. Steinbrunner, S. Friedowitz, R. R. Netz, and D. J. Bonthuis, "Transferable Ion Force Fields in Water from a Simultaneous Optimization of Ion Solvation and Ion-Ion Interaction," J. Phys. Chem. B 125, 85818587 (2021).

${ }^{66} \mathrm{~S}$. Cruz-León, W. Vanderlinden, P. Müller, T. Forster, G. Staudt, Y. Lin, J. Lipfert, and N. Schwierz, "Twisting DNA by Salt," bioRxiv2021.07.14.452306, 21-26 (2021). 